\title{
Macroglosia en Amiloidosis Primaria como Clave Diagnostica: Reporte de un Caso
}

\author{
Primary Amyloidosis with Macroglossia as Diagnostic Key: Case Reporte
}

\author{
Alvaro Vigouroux Valenzuela'; Francisca Donoso Hofer² \& Ana Verónica Ortega Pinto³
}

VIGOUROUX, V. A.; DONOSO, H. F. \& ORTEGA, P. A. V. Macroglosia en amiloidosis primaria como clave diagnostica: Reporte de un caso. Int. J. Odontostomat. 15(4):938-941, 2021.

RESUMEN: Amiloidosis se refiere a un grupo de enfermedades caracterizadas por el deposito extracelular de proteínas insolubles llamadas amiloide. La presentación intraoral de amiloidosis con macroglosia como signo primario es una entidad extraña, la cual puede afectar la funcionalidad de los pacientes aparte de la patología subyacente. Reportamos el caso de una paciente femenina de 85 años sin antecedentes mórbidos que al examen físico presenta macroglosia. La biopsia del tejido afectado fue estudiada bajo inmunohistoquimíca con tinción rojo congo, asociada al análisis de laboratorio que consignó un aumento en el componente monoclonal de cadenas livianas lambda. Se diagnosticó como amiloidosis primaria $(A L)$. Se considera fundamental el entrenamiento al odontólogo general para el diagnóstico precoz de estos signos clínicos, por la baja sobrevida de la amiloidosis y la probabilidad de patologías ocultas como mieloma múltiple, el cual está asociado en un $20 \%$ con AL.

PALABRAS CLAVE: macroglosia, amiloidosis.

\section{INTRODUCCIÓN}

Macroglosia se define como el aumento anormal en el tamaño de la lengua, clínicamente se presenta indolora con indentaciones en sus caras laterales y detritus alimenticio. Se categoriza en dos grandes grupos, macroglosia verdadera y pseudomacroglosia, la primera presente en patologías congénitas y adquiridas con hipertrofia real de tejido muscular y conectivo, generalmente causado por infiltrado proteico o glicógeno (Demirkan et al., 2017).

Su principal complicación es la disfagia, malnutrición, disartria y compresión de vías aéreas (Demirkan et al.; Oruba et al, 2018).

Dentro de las patologías adquiridas que pueden causar macroglosia se encuentra la amiloidosis, una rara condición presente en un gran espectro de enfermedades que tienen en común el deposito de agregado extracelular de proteínas llamado amiloide. Este material es insoluble y resistente a la proteólisis, se deposita principalmente en el tejido conectivo de distintos órganos, teniendo particular afinidad por el corazón, riñones, hígado y sistema nervioso (D'Aguanno et al., 2020).

La amiloidosis se presenta de manera localizada o sistémica, siendo mas común la amiloidosis de cadenas livianas (AL), entre el $10-20 \%$ de ésta se presenta con macroglosia (Mishra et al., 2018).

El diagnostico definitivo de AL se realiza con la biopsia del tejido afectado bajo tinción rojo congo (gold stándar ), la cual bajo luz birrefringente da un color verde manzana característico, la confirmación se da por presencia de cadenas livianas kappa o lambda en el análisis inmunohistoquímico (Melo Alves \& Marto, 2018).

\footnotetext{
${ }^{1}$ Cirujano Dentista, Pasante Hospital San Juan de Dios, Santiago, Chile.

${ }^{2}$ Servicio de Cirugía Maxilofacial - Hospital San Juan de Dios, Santiago, Chile.

${ }^{3}$ Patóloga Buco Maxilo Facial - Facultad de Odontología, Universidad de Chile, Santiago, Chile.
} 
La amiloidosis AL está asociada entre un 15-20 $\%$ a mieloma múltiple, generalmente en pacientes de la tercera edad, presentándose como primer signo tardío de la enfermedad (da Costa et al., 2018).

La expectativa de vida de estos pacientes desde la aparición de los síntomas varia entre 6 meses a 3 años aproximadamente, y su tratamiento consiste principalmente en quimioterapia (Williams et al., 2018).

\section{CASO CLÍNICO}

Se presentó al servicio de cirugía maxilofacial una paciente femenina de 85 años, marcha con aparato auxiliar (silla de ruedas). No presentaba antecedentes mórbidos. La paciente consultó por disfagia, dificultad para hablar, y baja de peso en los últimos meses.

Al examen físico se observó el aspecto de malnutrición, protrusión de la lengua y cuello en reloj de arena, depresible e indoloro, con función respiratoria conservada (Fig. 1).
Se efectuó el examen intraoral evidenciando desdentamiento parcial, macroglosia con protrusión labial, cierre labial forzoso. Detritus alimenticio en lengua con zonas depapiladas.

Bajo la sospecha de amiloidosis se realizó la biopsia de lengua y se solicitó hemograma completo.

El análisis histológico realizado con tinción rojocongo bajo luz polarizada confirmó el diagnóstico definitivo de amiloidosis (Fig. 2).

Los resultados de laboratorio de hemograma estaban dentro de los parámetros normales, electroforesis de proteínas en suero presentaba hipoalbuminemia con aumento de globulinas alfa-2 y beta. Inmunofijación en suero dio positivo a un aumento de componente monoclonal de cadena liviana lambda, sugerente de mieloma múltiple (MM).

Se derivó a la paciente para biopsia de médula para descartar MM, pero la paciente desistió del tratamiento solo con tratamientos paliativos.
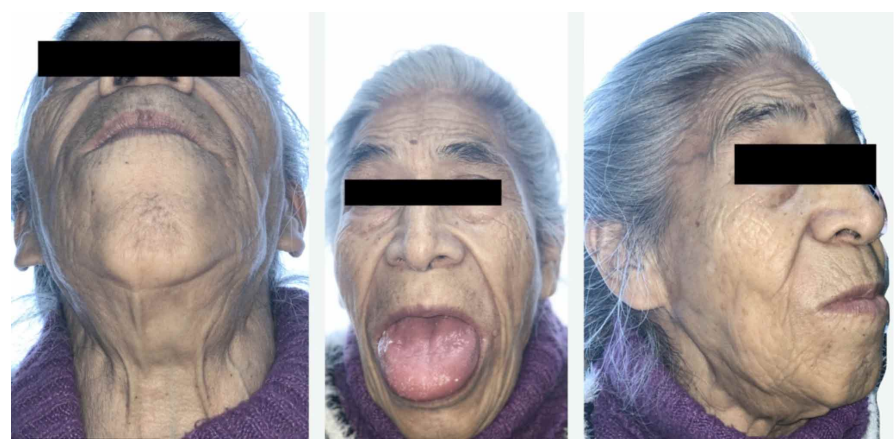

Fig. 1. Cuello en reloj de arena, Macroglosia y protrusión de lengua en reposo.
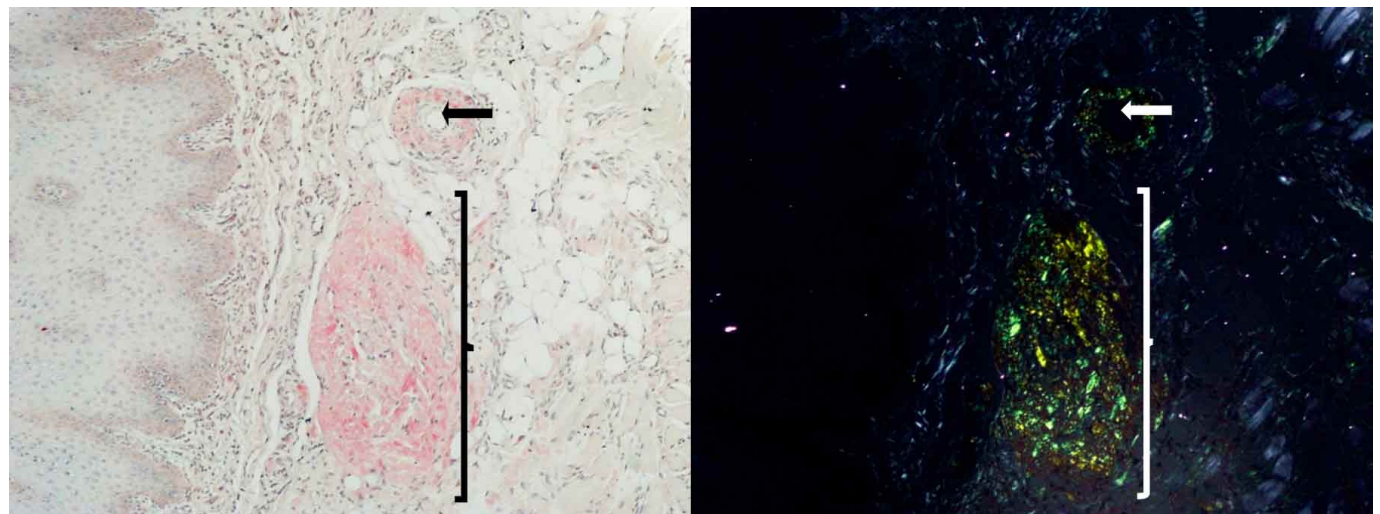

Fig. 2. a) Flecha negra: Amiloide en torno a un vaso sanguíneo.Corchete negro: Amiloide cercano a epitelio de revestimiento de mucosa oral. Tinción: Rojo Congo Luz tradicional. Aumento original: 100x. b) Flecha blanca: Amiloide en torno a un vaso sanguíneo. Corchete blanco: Amiloide cercano a epitelio de revestimiento de mucosa oral. Tinción: Rojo Congo Luz polarizada. Aumento original: 


\section{DISCUSIÓN}

La amiloidosis constituye un amplio espectro de enfermedades caracterizadas por la deposición extracelular de agregado fibrilar amorfo e insoluble, que para definirlo se le llamo amiloide.

El deposito de amiloide en diferentes órganos del cuerpo produce el daño celular lo cual conlleva la falla del tejido afectado. Es una patología degenerativa que aumenta con la edad y con factores ambientales, afecta principalmente al corazón, riñones, y al sistema nervioso (D'Aguanno et al.).

Existen numerosos tipos de amiloidosis dependientes de la proteína depositada, se dividen en genéticas y adquiridas, siendo de esta última la amiloidosis de cadenas livianas (AL) la mas común con un 65 de los casos. Su incidencia afecta entre 1 a 4.5 cada 100.000 personas al año (D'Aguanno et al.).

El diagnostico definitivo se hace con la inmunohistoquimica, tinción rojo congo al microscopio bajo luz polarizada, lo que da su característico birrefringencia color verde manzana (MaturanaRamírez et al., 2018).

La mayor causa de morbilidad y mortalidad es la cardiomiopatía, la cual a los 5 años presenta una mortalidad menor al $10 \%$ de los pacientes, generalmente por falla ventricular (Magesh et al., 2017).

El principio de tratamiento para la amiloidosis $A L$ se basa en quimioterapia enfocada en las discrasias de células plasmáticas lo que apunta a reducir la producción de las cadenas livianas amiloides limitando el daño en los órganos. También se realiza trasplante autólogo de células madres (ASCT) e inmunoterapia (Wechalekar et al., 2016).

La amiloidosis AL tiene una predominancia por el sexo masculino, edad promedio de diagnostico es de 65 años, solo el $10 \%$ de los pacientes es diagnosticado bajo los 50 años. El $20 \%$ de los pacientes con AL presentan mieloma múltiple (Hsiao et al., 2019).

La macroglosia se presenta en un 10-20\% de los casos de AL y puede aparecer como signo único de la enfermedad, por lo que es fundamental su diagnostico diferencial con otras macroglosias de origen tumorales, malformaciones neovasculares, asociada a trastornos hereditarios, hipotiroidismo, deficiencia de vitamina B12 y pseudomacroglosias (Sun et al., 2017; Shahbaz et al., 2018).

La presentación de AL con macroglosia como signo primario es una rara entidad, y en nuestro caso en particular solo fue tratada de manera paliativa, ya que ala espera de una biopsia de medula para descartar mieloma múltiple la paciente desistió del tratamiento.

\section{CONCLUSIÓN}

La baja sobrevida de la amiloidosis primaria hace fundamental el diagnostico diferencial precoz para un tratamiento oportuno, es vital el entrenamiento de cirujanos dentistas para la pesquisa de signos intraorales evidentes como la macroglosia que puede esconder patologías asociadas tan severas como el mieloma múltiple.

VIGOUROUX, V. A.; DONOSO, H. F. \& ORTEGA, P. A. V. Primary amyloidosis with macroglossia as diagnostic key: Case report. Int. J. Odontostomat., 15(4):938-941, 2021.

ABSTRACT: Amyloidosis groups a large set of diseases characterized by the deposit of an extracellular insoluble protein known as amyloid. Intraoral presentation of macroglossia induced by amyloidosis is a rare entity that can impair patient function and hide other pathological conditions. We report a case of an 85-year-old woman with no morbid background were the physical examination revealed macroglossia. Primary systemic amyloidosis (AL) definitive diagnosis was made after the tongue biopsy resulted positive under congo red histochemical staining, and serum immunoelectrophoresis had elevated levels for lambda light chains. Primary dental dentistry training on semiotics is imperative to diagnose early stages of hidden pathologies with low life expectancy such as AL, which in $20 \%$ of cases are associated with life threatening diseases like multiple myeloma.

KEY WORDS: macroglossia, amyloidosis.

\section{REFERENCIAS BIBLIOGRÁFICAS}

D'Aguanno, V.; Ralli, M.; Artico, M.; Russo, F. Y.; Scarpa, A.; Fiore, M.; Tirassa, P.; Severini, C.; de Vincentiis, M. \& Greco, A. Systemic amyloidosis: a contemporary overview. Clin. Rev. Allergy Immunol., 59(3):304-22, 2020. 
da Costa, K. V. T.; Ribeiro, C. M. B.; de Carvalho Ferreira, D.; Gonçalves, L. S.; de Almeida, O. P.; de Carvalho Silva, L. T.; Bastos, Y. V. P. \& Ferreira, S. M. S. Dysphagia due to macroglossia in a patient with amyloidosis associated with multiple myeloma: A case report. Spec. Care Dentist., 38(4):2558, 2018.

Demirkan, S.; S,avk, E.; Alp, A.; Doger, F. K.; Kadikoylu, G. \& Gunduz, O. Macroglossia as a presenting feature of multiple myeloma. $J$. Family Med. Prim Care., 6(1):146-7, 2017.

Hsiao, P. J.; Chang, Y. C.; Tsao, Y. H.; Wu, K. L.; Kao, Y. H.; Chan, J. S.; Wang, C. H.; Lin, Y. Y.; Chuu, C. P. \& Lin, Y. S. Ptosis and macroglossia in a woman with systemic light-chain amyloidosis. Clin. Chim. Acta, 494:112-5, 2019.

Magesh, B.; Kadeli, D.; Bohra, S.; Krishnaprasath, V. \& Keshava, R. Cardiac amyloidosis, an infiltrative heart disease presenting as arrhythmia-a case report. J. Clin. Diagn Res., 11(4):OD14-OD15, 2017.

Maturana-Ramírez, A.; Ortega, A. V.; Labbé, F. C.; de Moraes, Ê. \& Aitken-Saavedra, J. P. Macroglossia, the first manifestation of systemic amyloidosis associated with multiple myeloma: Case report. J. Stomatol. Oral. Maxillofac. Surg., 119(6):514-7, 2018.

Melo Alves, J. \& Marto, N. Macroglossia in light-chain amyloidosis. N. Engl. J. Med., 378(24): 2321, 2018.

Mishra, K.; Jandial, A.; Prakash, G. \& Malhotra, P. Macroglossia and amyloidosis. QJM, 111(11):835-6, 2018.

Oruba, Z.; Kaczmarzyk, T.; Urban'czyk, K.; Jurczyszyn, A.; Fornagiel, S.; Ga?a_zka, K.; Bednarczyk, A. \& Chomyszyn-Gajewska, M. Intraoral manifestation of systemic $A L$ amyloidosis with unique microscopic presentation of intracellular amyloid deposition in striated muscles. Pol. J. Pathol., 69(2):200-4, 2018.

Shahbaz, A.; Aziz, K.; Umair, M.; Malik, Z. R.; Awan, S. I. \& Sachmechi, I. Amyloidosis presenting with macroglossia. Cureus., 10(8):e3185, 2018.

Sun, L.; Zhang, L.; Hu, W.; Li, T. F. \& Liu, S. Case report: One case of primary $\mathrm{AL}$ amyloidosis repeatedly misdiagnosed as scleroderma. Medicine, 96(50):e8771, 2017.

Wechalekar, A. D.; Gillmore, J. D. \& Hawkins, P. N. Systemic amyloidosis. Lancet, 387(10038):2641.2654, 2016.

Williams, M. U.; Murphy, C. E.; Gore, R. S. \& Fentanes, E. Lingual liability: macroglossia and dyspnoea as the harbinger of systemic $\mathrm{AL}$ (light-chain) cardiac amyloidosis. BMJ Case Rep., 11(1):e225923, 2018.

\author{
Dirección para correspondencia: \\ Dr. Alvaro Vigouroux \\ Cirujano Dentista \\ Pasante Hospital San Juan de Dios \\ Santiago \\ CHILE
}

E-mail: a.vigouroux@hotmail.com 